\title{
Compatibility analysis of reactive dyes by exhaustion-fixation and adsorption isotherm on knitted cotton fabric
}

\author{
Abu Naser Md Ahsanul Haque ${ }^{1 *}$ MA Hannan ${ }^{2}$ and Md Masud Rana ${ }^{3}$
}

\author{
* Correspondence: \\ naser.te@daffodilvarsity.edu.bd \\ ${ }^{1}$ Daffodil International University, \\ Dhaka, Bangladesh \\ Full list of author information is \\ available at the end of the article
}

\begin{abstract}
Compatibility analysis was done by exhaustion, fixation and adsorption isotherm upon three reactive dyes; Remazol Red RR, Remazol Yellow RR and Remazol Blue RR. The known solutions of each dye were tested in UV-visible spectrophotometer and the extinction coefficients were calculated by drawing absorbance versus concentration curve. Dyeing processes were carried out in 4 different dye concentrations $(0.5 \%, 1 \%$, $2 \%$ and $3 \%$ ) for each of the dye. The post dye and post wash liquors were tested in UV-visible spectrophotometer and the dye concentrations of those solutions were calculated through the Beer-Lambert law. The exhaustion\%, fixation\% of all three dyes was compared between them and their adsorption isotherm models were drawn. In between Langmuir and Freundlich isotherm model, the last one had the supremacy. Among the dyes, Blue RR had better exhaustion performance although it was not pleasingly attached with the fiber during fixation. Red RR was excellent in both exhaustion and fixation stages. It was found that in deeper concentrations the compatibility among these three dyes would be low. But experimental data shows that introducing more electrolytes and reducing the temperature as well as the alkali amount can improve these dyes' compatibility.
\end{abstract}

Keywords: Exhaustion; Fixation; Adsorption isotherm; Compatibility

\section{Introduction}

Reactive dye introduced on 1956 and for the first time dyeing became possible by direct chemical linkage between dye and fiber (Shenai, 1993). But all classes of reactive dye do not react in the same manner. So the group of dyes used for a ternary shade should have compatibility among themselves. Importantly, reactive dyes in a mixture should all exhaust and react with the fiber at about the same rate so that the shade builds up accurately. Dyes which are from different ranges, with different reactive groups, should not be used together because of their different dyeing character and reactivity.

Compatible dyeing performance requires careful control of the dyeing parameters such as temperature, salt and alkali concentrations, the dyeing time and the liquor ratio. There is often a doubt about the particular reactive group presents in a reactive dye. For that reason in most of the cases selection of dyes depends on the maker's recommendations (Broadbent, 2001).

\section{Springer}

C 2015 Haque; licensee Springer. This is an Open Access article distributed under the terms of the Creative Commons Attribution License (http://creativecommons.org/licenses/by/4.0), which permits unrestricted use, distribution, and reproduction in any medium, provided the original work is properly credited. 
Shenai (1997) discussed in detail about the chemistry of vinyl sulphone dyes like Remazol class. Common salt and alkali plays the vital role in exhaustion and fixation of these dyes and addition of salt to the dye bath before adding the alkali is also essential. In reactive dyeing, though water is the competitor for reaction with the dye, cellulose fiber takes part in the reaction in majority. Because the substantivity of reactive dye to the fiber is greater than that to water (Chinta and Vijaykumar 2013).

But factually all the reactive dyes do not have the same range of substantivity and reactivity. Reactivity is compulsory for these dyes but higher reactivity of a dye can spoil the dyeing due to hydrolysis. So the compatibility of the dyes used for ternary shades should be analyzed carefully to make the maximum utilization of each dyestuff especially when the reactive groups in them are different.

\section{Literature review}

Fowler (1997) had written about a tri-chromatic system where the dyestuffs were Remazol Red RR, Remazol Blue RR and Remazol Yellow RR. He confirmed that all three dyes contain at least one vinyl sulphone group as their reactive group. In his article he had not described about any of his testing procedures though gave his results. The fixation yields he had got was $83 \%$ for Red RR, $81 \%$ for Blue RR and $79 \%$ for Yellow RR. He declared that this RR combination can meet all requirements with high cost effectiveness; which is actually a meaning of compatibility between the dyes. Because one of the meanings of high cost effectiveness is utilizing each of the dye at highest possible percentage in a equal period of time.

But Sultana and Uddin (2007) tested the compatibility of same three dyes by calculating their dye extinction coefficient, exhaustion and fixation percentages. They mentioned that in a particular time if the exhaustion or fixation percentages of three dyes do not superimpose each other they cannot be said as compatible. In their experiment they got different dye extinction coefficients; particularly that value for Red RR was quite high from the other two. And the fixation of Red RR was also had a significant difference from the others (Red RR 91.89\%, Blue RR 77.48\%, Yellow RR 64.20\%). So they decided against the compatibility of these three dyes.

Moreover, According to Cay et al. (2007) those three dyes are containing different reactive groups! Remazol Red RR is a hetero bi-functional dye which contains both the monochlorotriazine and vinyl sulphone group (MCT-VS). Blue RR is homo bifunctional containing two vinyl sulphone groups (VS-VS) and Yellow RR, a mono functional dye contains only a vinyl sulphone group (VS).

The behavior of bi-functional dyes was studied by several researchers. Dalal and Desai (1996) studied on some bi functional reactive dyes to find out their dyeing capability. The dyes showed medium to good substantivity which was detected by their exhaustion and fixation study. Son et al. (2005) applied four hetero bi-functional reactive dyes to nylon fibers using various $\mathrm{pH}$ and temperature conditions. They found that hetero bi-functional reactive dyes afford great chance for efficient reaction between dye and fiber due to the mixed double-anchors. Besides, Ali et al. (2012) worked with MCT-VS, VS and MCT based reactive dye and found in comparison that MCT-VS dye had better color strength than MCT dye. And a VS dye had better color strength than MCT-VS dye.

There were some modeling of adsorption isotherm systems, both for two parameters and three parameters isotherm in the article of Foo and Hameed (2010). In two 
parameters they discussed with Langmuir, Freundlich, Tempkin, Dubinin-Radushkevich, Flory-Huggins and Hill isotherm model. And according to Choudhury (2006), not all the models but dyeing on cellulose fiber with reactive dye generally follows Langmuir and Freundlich model of adsorption isotherm. And in recent, Ara et al. (2013) studied with Remazol Red RR adsorption to activated carbon. They analyzed both Langmuir and Freundlich isotherm model and observed that the Freundlich isotherm was fitted with the adsorption behavior of Red RR.

As Remazol RR combination have different reactive group (Cay et al., 2007) and Sultana and Uddin (2007) questioned about their compatibility, further analysis was necessary about these; may be from a different angle. For that reason this research had analyzed the compatibility not only by exhaustion and fixation, but also with their adsorption isotherm to understand their individual interaction with the fiber. Adsorption isotherm analysis of reactive dye has been discussed by many researchers earlier, but this is the first time the adsorption isotherm was considered to explain the compatibility among three reactive dyes used for ternary shades. The Least square method (Cantrell, 2008) was applied in this research to calculate the slope and y intercept of the isotherm models. And according to Taylor (1990), coefficient of determination, $r^{2}$ is much meaningful in graph explanation. So that was considered in this research to explain the fitting of the adsorption isotherm models.

\section{Method}

\section{Fabric and dyes}

In this research work used single jersey scoured and bleached cotton fabric was supplied by Impress-Newtex Composite Textile Limited, Bangladesh. It was manufactured from $26 \mathrm{Ne}$ combed yarn and stitch length was found $2.62 \mathrm{~mm}$ with a GSM (gram per square meter) of 160. Remazol Red RR (MCT-VS dye), Remazol Yellow RR (VS dye), Remazol Blue RR (VS-VS dye) was used for the dyeing of fabric purchased from DyStar, Germany by Impress-Newtex Composite Textile Limited, Bangladesh (Figures 1, 2 and 3).

\section{Measurement of dye extinction coefficient}

The absorbance of the dye solutions were measured by using an UV-Visible spectroscopy instrument (UV1800). To calculate the dye extinction coefficient, 5 solutions of different known concentrations ( $5 \mathrm{mg} / \mathrm{L}, 10 \mathrm{mg} / \mathrm{L}, 20 \mathrm{mg} / \mathrm{L}, 30 \mathrm{mg} / \mathrm{L}, 50 \mathrm{mg} / \mathrm{L}$ ) were considered for each of the dye. They were tested one by one in the instrument and absorbance versus wavelength curves was obtained. Each curve showed a same pick point for different concentrations of a particular dye. That was the $\lambda_{\max }$ value for the

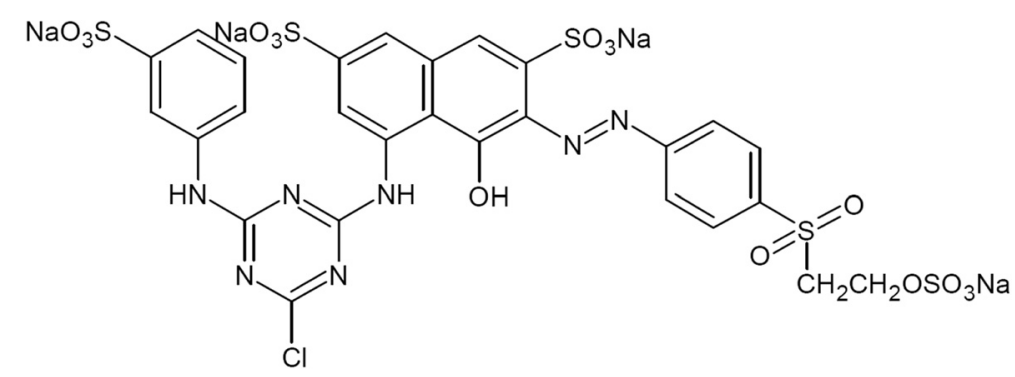

Figure 1 Chemical structure of a MCT-VS dye. 


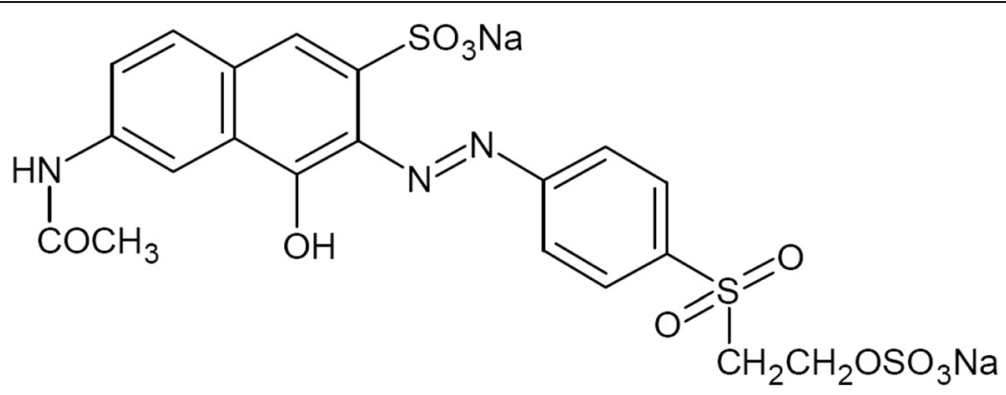

Figure 2 Chemical structure of a VS dye.

particular dye and was $420 \mathrm{~nm}$ for Yellow RR, $517 \mathrm{~nm}$ for Remazol Red RR and $587 \mathrm{~nm}$ for Remazol Blue RR. The absorbance values of the dyes at $\lambda_{\text {max }}$ points were considered for calculations. From the Beer-Lambert law-

$$
A=\varepsilon \times l \times c
$$

Where, $A=$ Absorbance, $\varepsilon=$ Dye extinction coefficient $\left(\mathrm{L} \mathrm{mg}^{-1} \mathrm{~cm}^{-1}\right), l=$ length $(\mathrm{cm})$ of solution the light passes through and $c=$ concentration of solution (mg/liter). From above equation-

$$
\varepsilon \times l=\frac{A}{c}
$$

The values were recorded from the instrument, and a graph was drawn by plotting the concentration values in $\mathrm{x}$-axis and absorbance values in $\mathrm{y}$-axis. According to equation (2) the slope of the graph was $\varepsilon \times l$. For our instrument, the value of $l$ was $1 \mathrm{~cm}$ constant. So, the slope of the graph actually equaled to the dye extinction coefficient $(\varepsilon \times 1=\varepsilon)$ and was calculated by least square method.

\section{Dyeing and after-treatment}

Dyeing processes were carried out in four different dye concentrations $(0.5 \%, 1 \%, 2 \%$ and 3\%). Furthermore, three different self shades (Red, Yellow and Blue) were created on fabric. The weight of fabric of each sample was kept constant during dyeing and same dyeing parameters were maintained for each shade percentage. The recipe were followed in the dyeing process are presented in Table 1.

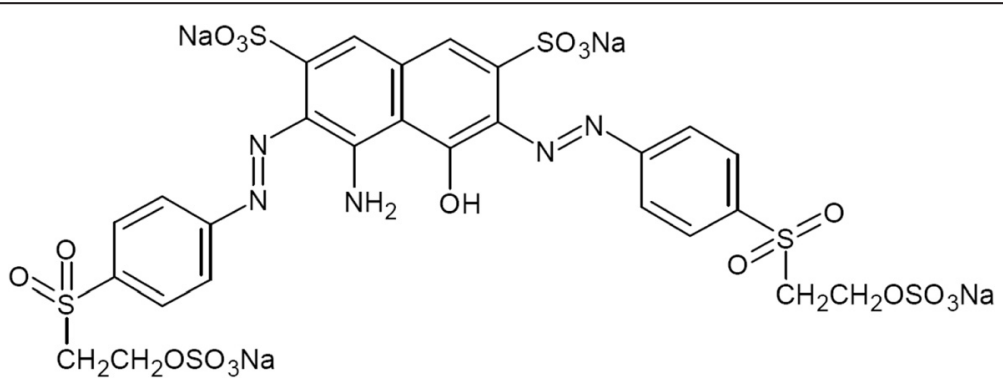

Figure 3 Chemical structure of a VS-VS dye. 
Table 1 Recipe for dyeing

\begin{tabular}{lllll}
\hline $\begin{array}{l}\text { Chemical and } \\
\text { parameter }\end{array}$ & \multicolumn{4}{l}{ Shade \% OWF (on the weight of fabric) } \\
\cline { 2 - 5 } & $\mathbf{0 . 5 \%}$ & $\mathbf{1 . 0 \%}$ & $\mathbf{2 . 0 \%}$ & $\mathbf{3 . 0 \%}$ \\
\hline $\mathrm{NaCl}$ & $30 \mathrm{~g} / \mathrm{L}$ & $50 \mathrm{~g} / \mathrm{L}$ & $50 \mathrm{~g} / \mathrm{L}$ & $60 \mathrm{~g} / \mathrm{L}$ \\
$\mathrm{Na}_{2} \mathrm{CO}_{3}$ & $5 \mathrm{~g} / \mathrm{L}$ & $5 \mathrm{~g} / \mathrm{L}$ & $5 \mathrm{~g} / \mathrm{L}$ & $5 \mathrm{~g} / \mathrm{L}$ \\
$\mathrm{NaOH}$ & $0 \mathrm{~g} / \mathrm{L}$ & $0.3 \mathrm{~g} / \mathrm{L}$ & $0.3 \mathrm{~g} / \mathrm{L}$ & $0.45 \mathrm{~g} / \mathrm{l}$ \\
$\mathrm{Fabric} \mathrm{weight}$ & $5 \mathrm{gram}$ & $5 \mathrm{gram}$ & $5 \mathrm{gram}$ & $5 \mathrm{gram}$ \\
$\mathrm{M}: \mathrm{L}$ & $1: 8$ & $1: 8$ & $1: 8$ & $1: 8$ \\
Temperature & $60^{\circ} \mathrm{C}$ & $60^{\circ} \mathrm{C}$ & $60^{\circ} \mathrm{C}$ & $60^{\circ} \mathrm{C}$ \\
Time & 60 minutes & 60 minutes & 60 minutes & 60 minutes \\
\hline
\end{tabular}

Dyeing of all samples was performed in a Mathis Labomat lab dyeing machine, using 12 sealed stainless steel dye pots. First, each individual samples and required amount of water were taken into the dye pot and ran the Lab dyeing machine. The flow chart of dyeing procedure can be shown as-

Sample and liquor loaded on machine

$\downarrow$

Raising temperature to $60^{\circ} \mathrm{C}$

$\downarrow$

Salt addition; run 2 minutes

$\downarrow$

Dye addition; run 2 minutes

$\downarrow$

Alkali addition; run 60 minutes

$\downarrow$

Fabric taken out for after treatment

The after-treatment was done in the following order, Hot wash $\left(60^{\circ} \mathrm{C}\right) \rightarrow$ Cold wash $\left(28^{\circ} \mathrm{C}\right) \rightarrow$ Squeezing $\rightarrow$ Drying. Each fabric was washed in $300 \mathrm{ml}$ water for five minutes and then taken out to for squeezing. The post wash liquor of each stage was preserved in containers for the UV-Visible spectroscopy test. Finally, dyed washed samples were dried in dryer for 30 minutes at $60^{\circ} \mathrm{C}$.

\section{Analysis of post dye and post wash liquors}

The post dye liquors, post hot wash liquors and post cold wash liquors were tested one by one in UV1800. From equation (1)-

$$
c=\frac{A}{\varepsilon \times l}
$$

By putting the values of $\varepsilon, l$ and $A$, the dye concentration, $c$ of the liquors were found in $\mathrm{mg} / \mathrm{L}$.

The amount of dye, $d(\mathrm{mg})$ in the post dye and post wash liquors were calculated by the following equation-

$$
d=c \times V
$$


Where, $V$ is the volume of the liquor (liter). After getting the amount of dye in the liquors, exhaustion percentage (E\%) and fixation percentage (F\%) were calculated by following formula-

$$
E^{\%}=\frac{D_{i}-D_{p d l}}{D_{i}} \times 100
$$

Where, $D_{i}=$ Initial dye weight in dye bath $(\mathrm{mg})$ and $D_{p d l}=$ dye weight $(\mathrm{mg})$ in post dye liquor.

$$
F \%=\frac{D_{i}-D_{p d l}-D_{p h l}-D_{p c l}}{D_{i}} \times 100
$$

Where, $D_{i}=$ Initial dye weight $(\mathrm{mg}), D_{p d l}=$ dye weight $(\mathrm{mg})$ in post dye liquor, $D_{p h l}=$ dye weight $(\mathrm{mg})$ in post hot wash liquor and $D_{p c l}=$ dye weight $(\mathrm{mg})$ in post cold wash liquor. The loss of dye (L\%) in hot wash and cold wash were calculated by using the formula-

$$
L \%=\frac{D}{D_{i}} \times 100
$$

Where, $D$ is the weight of dye (mg) in post hot wash or post cold wash liquor and $D_{i}=$ Initial dye weight in dye bath (mg).

\section{Adsorption isotherm analysis}

Dyeing on cellulose fiber with reactive dye commonly fitted to the Langmuir model (8) and the Freundlich model (9).

$$
\begin{aligned}
& \frac{C_{f}}{C_{\text {max }}}=\frac{b C_{s}}{1+b C_{s}} \\
& C_{f}=k C_{s}^{(1 / n)}
\end{aligned}
$$

Where, $C_{f}=$ amount of dye adsorbed on the adsorbent $(\mathrm{mg} / \mathrm{g}), C_{s}=$ dye concentration at equilibrium $(\mathrm{mg} / \mathrm{L}), b=$ Energy of the adsorption, $C_{\max }=$ Maximum number of adsorption sites, $n=$ Heterogeneity level and $k=$ Adsorption capacity. These models can be rearranged into the linear form as follows-

$$
\begin{aligned}
& \frac{C_{s}}{C_{\mathrm{f}}}=\frac{C_{s}}{C_{\max }}+\frac{1}{b C_{\max }} \\
& \log \left(C_{f}\right)=\log (k)+(1 / n) \log \left(C_{s}\right)
\end{aligned}
$$

The graphs of $C_{s} / C_{f}$ against $C_{s}$ (Langmuir model) and $\log \left(C_{f}\right)$ against $\log \left(C_{s}\right)$ (Freundlich model) were drawn. The best fit slope $\left(1 / b C_{\max }\right.$ and $\left.1 / n\right)$, and the intercept $\left[1 / C_{\text {max }}\right.$ and $\log (k)]$ of these curves were determined by using the formula of least squares method. The coefficient of determination, $r^{2}$ was calculated by the following formula-

$$
r^{2}=\left[\frac{m \sum\left(x_{i} y_{i}\right)-\sum x_{i} \sum y_{i}}{\sqrt{\left\{m \sum x_{i}^{2}-\left(\sum x_{i}\right)^{2}\right\}}\left\{m \sum y_{i}^{2}-\left(\sum y_{i}\right)^{2}\right\}}\right]^{2}
$$


Table 2 Calculated extinction coefficient of the dyes

\begin{tabular}{|c|c|}
\hline Dye & Dye extinction coefficient $\left(\mathrm{L} \mathrm{mg}^{-1} \mathrm{~cm}^{-1}\right)$ \\
\hline Remazol Red RR & 0.0158 \\
\hline Remazol Yellow RR & 0.0097 \\
\hline Remazol Blue RR & 0.0049 \\
\hline
\end{tabular}

Where, $m$ is the number of pairs of data; $x$ and $y$ are the $\mathrm{x}$-coordinates and $\mathrm{y}$-coordinates. The $r^{2}$ is the ratio of the explained variation to the total variation. It is such that $0 \leq r^{2} \leq 1$, and denotes the strength of the linear association between $x$ and $y$. It represents the percent of data that is closest to the line of best fit. For example, if $r^{2}=0.850$, which means that $85 \%$ of the total variation in $y$ can be explained by the linear relationship between $x$ and $y$. The other $15 \%$ of the total variation in $y$ remains unexplained.

\section{Results and discussion}

\section{Dye extinction coefficients}

The dye extinction coefficient found for all three dyes are listed in Table 2. The table shows the dye extinction coefficient order as Red RR $>$ Yellow RR $>$ Blue RR.

\section{Exhaustion properties of the dyes}

For this analysis, first the shade percentages are plotted in $\mathrm{x}$-axis and dye take up or exhaustion percentages are plotted in y-axis (Figure 4). For the same shade percentage of dye, three dyes deviate in dye take up with fiber. The result shows that Blue RR and Red RR always had a higher and almost similar percentage of exhaustion. But yellow RR had a poor exhaustion percentage in comparison with the others especially in deeper shade percentages.

Reactivity of VS group is higher than MCT group. As Yellow RR is a mono functional dye with one VS group; Blue RR is a homo bi-functional dye with two VS group. Blue RR had a higher exhaustion than Yellow RR as it doubled the chance of dye molecules to be attracted. On the other hand Red RR is a hetero bi-functional dye. It has two

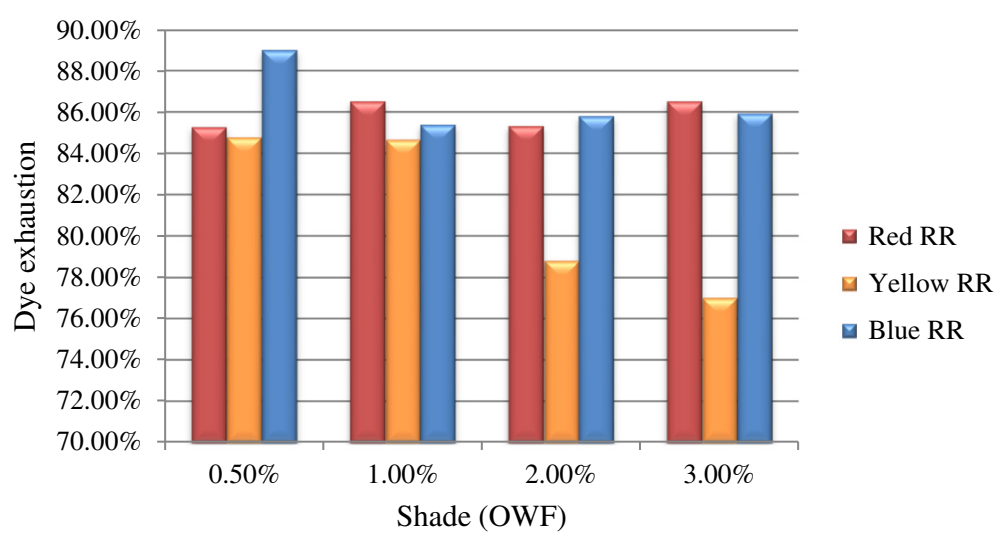

Figure 4 Comparison in exhaustion properties of dyes. 


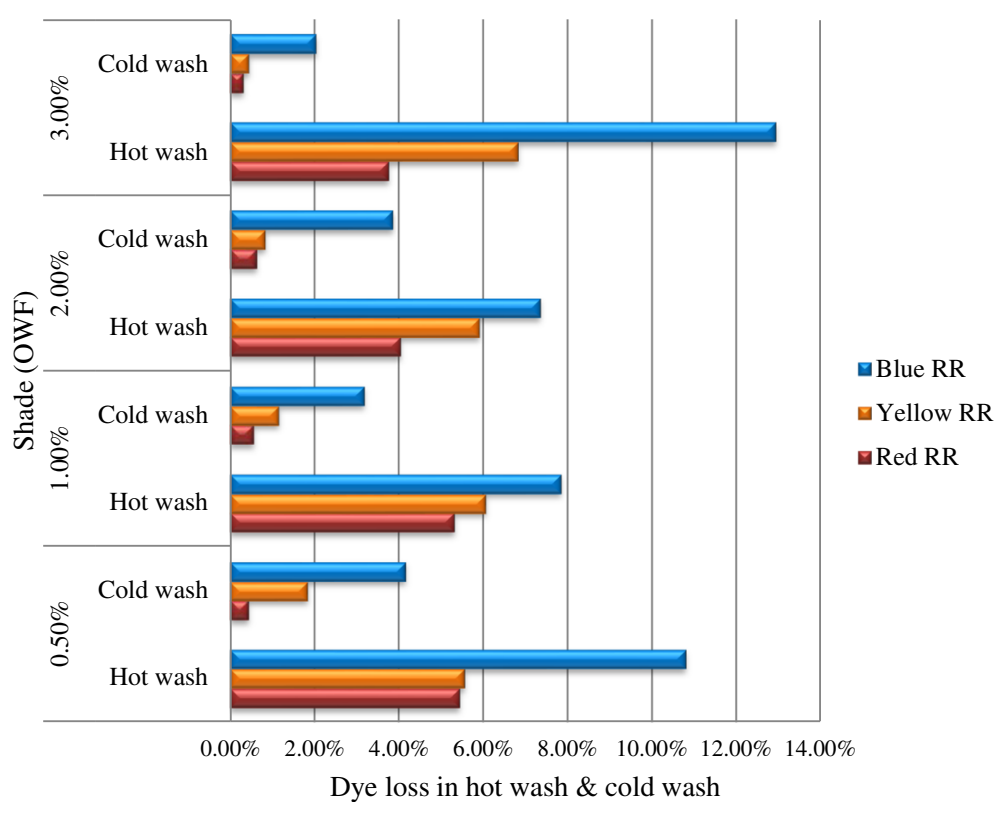

Figure 5 Comparison in dye loss by hot wash and cold wash.

different groups; one VS another MCT. More reactive groups made the better chance for its affinity towards fiber.

\section{Dye loss in washing}

This comparison is done by plotting the $L \%$ in $x$-axis for each dyeing and shade\% of dye in y-axis. The graph (Figure 5) shows that the Blue RR was always having a greater loss in washing in each concentration and in maximum cases Yellow RR had greater tendency to wash away than the Red RR.

As Blue RR has double VS group, it has higher reactivity than the other two dyes. So the possibility of hydrolysis of this dye is also high than other two. And in between Yellow RR

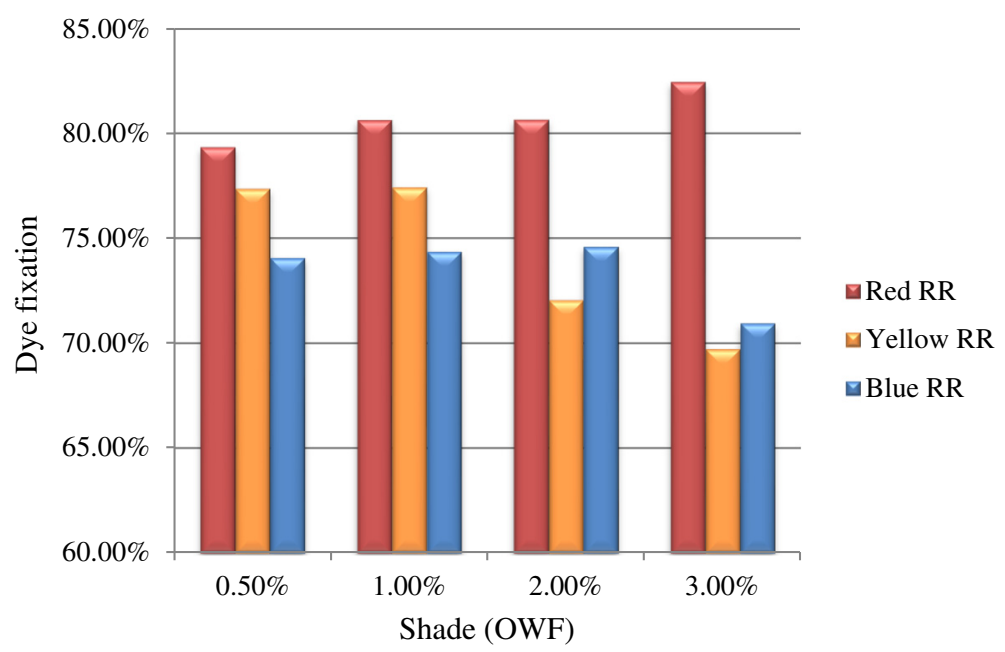

Figure 6 Comparison in fixation properties of the dyes. 


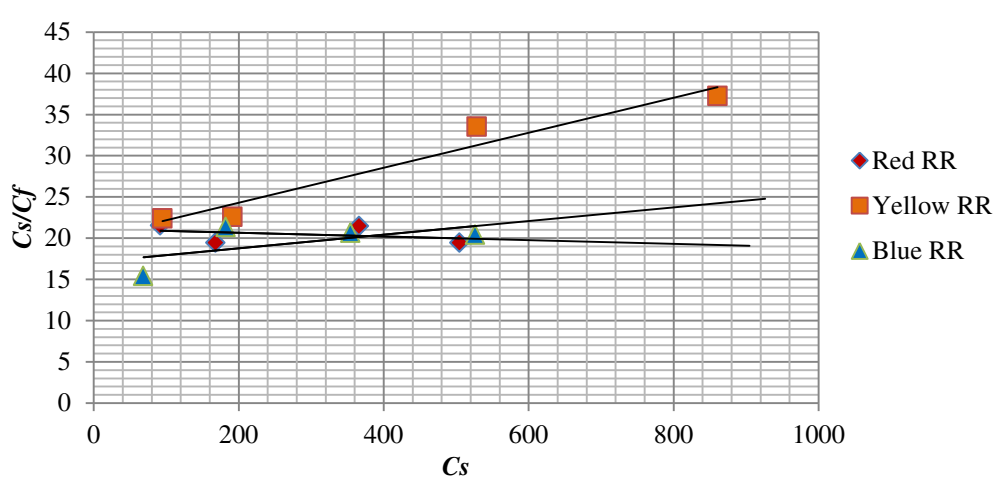

Figure 7 Langmuir adsorption isotherm model.

and Red RR, Red RR had a lower loss in washing as it has VS group as well as a MCT group also in the same molecule. MCT group has lower reactivity and so lower tendency for dye hydrolysis.

\section{Fixation properties of the dyes}

To compare the fixation of the three dyes, the shade\% is taken in $\mathrm{x}$-axis, and the fixation\% of dye in fabric is plotted in y-axis (Figure 6).

The graph is clearly different from exhaustion graph. In fixation, Red RR was obviously best among these three. In deeper concentrations, it was far better. Other two dyes Yellow RR and Blue RR were the competitor among themselves. Fixation performance of Yellow RR was slightly good in comparison with Blue RR in lower concentrations but at deeper concentration it was less than Blue RR.

As Red RR has a MCT group with VS group, because of that it had shown its reactivity in a controlled manner than the others. So it faced less hydrolysis problem and the dye exhausted in the fabric was fixed almost. But the other two dyes, having VS and double VS groups, were not fixed as like as Red RR because of their high reactivity which let them reacts with water.

\section{Adsorption isotherm analysis}

Diagrams in Figure 7 represent the interpretation of Langmuir adsorption isotherm for Remazol Red RR, Yellow RR and Blue RR; showing the parameters dependence $\left(C_{s} / C_{f}\right)$ in relation to the equilibrium dye concentration $\left(C_{s}\right)$ of the dye bath.

In Table 3, analytical formulations of Langmuir isotherms are shown, Langmuir parameters $C_{\text {max }}$ and $b$, as well as values of coefficient of determination $r^{2}$ for Langmuir diagram. Determination coefficient is the relative measurement of representativeness of

Table 3 Analytical illustration of Langmuir isotherm with coefficients

\begin{tabular}{lllll}
\hline Dyes & $\begin{array}{l}\text { Analytical expression } \\
\text { of curve (Langmuir } \\
\text { equation) }\end{array}$ & \multicolumn{2}{l}{ Langmuir parameters } & $r^{2}$ \\
\cline { 4 - 5 } $\boldsymbol{C}_{\max }(\mathbf{m g} \mathbf{g})$ & $\boldsymbol{b}(\mathbf{L} / \mathbf{m g})$ & \\
\hline Remazol Red RR & $C_{s} / C_{f}=-0.0022 C_{s}+21.1$ & -454.545 & -0.0001 & 0.122 \\
Remazol Yellow RR & $C_{s} / C_{f}=0.0212 C_{s}+20.042$ & 47.17 & 0.00106 & 0.9499 \\
Remazol Blue RR & $C_{s} / C_{f}=0.0083 C_{s}+17.123$ & 120.482 & 0.00048 & 0.3677 \\
\hline
\end{tabular}




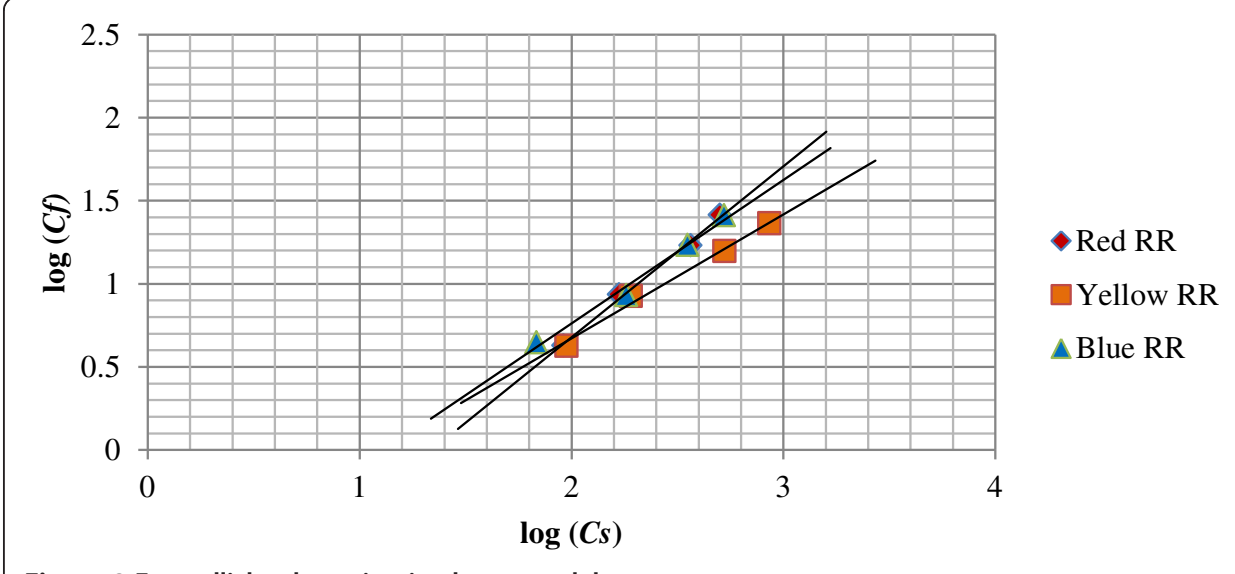

Figure 8 Freundlich adsorption isotherm model.

regressive line which measures of model's utility. In the cases when $r^{2}=1$, the model is functional or linear, i.e. all points in dispersion diagram are in the regression line. From Table 3 for determination coefficient, it is noticeable that the processes with Red RR and Blue RR have lower values (0.122 and 0.3677). So, Langmuir model does not cover in an acceptable way experimental data for these two dyes. Moreover, the negative values for Langmuir isotherm constants for Red RR adsorption indicate the inadequacy of the isotherm model to explain the adsorption process. So compatibility analysis of these dyes is not possible by this model.

Diagrams in Figure 8 represent Freundlich model for adsorption of those dyes on fiber, which was plotted by $\log \left(C_{s}\right)$ and $\log \left(C_{f}\right)$ values in $\mathrm{x}$-axis and $\mathrm{y}$-axis respectively. According to these diagrams, the high functionality of variables is observed, which not the case in Langmuir model was. Based on these diagrams, Freundlich invariables were determined and by applying them, the suitability of the model for describing adsorption process of applied dye on cotton fabric was determined. Table 4 shows the results connected to Freundlich isotherm adsorption model. $k$, one of the Freundlich constants is used as the relative measurement of the adsorption capacity, the higher $k$ implies on higher level of adsorption capacity. This value was found higher for Yellow RR which indicates its better capacity of adsorption.

The second Freundlich constant, $n$, is the parameter that alters with heterogeneity level. It refers to the distribution of bonded dye molecules on the fiber surface. It is the inverse of Freundlich model slope. High $n$ value indicates higher heterogeneity level, If $1 / n$ is above one, it is an indication of cooperative adsorption. When the $1 / n$ is close to zero it indicates more heterogeneous surface. In this case, invariable $n$ has highest value for Yellow RR. It indicates its heterogeneity in the adsorbent surface for which it had

Table 4 Analytical illustration of Freundlich isotherm with coefficients

\begin{tabular}{|c|c|c|c|c|}
\hline \multirow[t]{2}{*}{ Dyes } & \multirow{2}{*}{$\begin{array}{l}\text { Analytical expression of } \\
\text { curve (Freundlich equation) }\end{array}$} & \multicolumn{2}{|c|}{ Freundlich parameters } & \multirow[t]{2}{*}{$r^{2}$} \\
\hline & & $k$ & $n$ & \\
\hline Remazol Red RR & $C_{f}=0.042 C_{s}^{1 / 0.973}$ & 0.042 & 0.973 & 0.9953 \\
\hline Remazol Yellow RR & $C_{f}=0.153 C_{s}^{1 / 1.343}$ & 0.153 & 1.343 & 0.9902 \\
\hline Remazol Blue RR & $C_{f}=0.108 C_{s}^{1 / 1.158}$ & 0.108 & 1.158 & 0.9864 \\
\hline
\end{tabular}


not exhaust well in result though it had a better adsorption capacity. Red RR had shown cooperative as well as low heterogeneity level.

Determination coefficient of Freundlich isotherms, at all examined conditions, are far from those gained for Langmuir diagram. It reached the value of almost 1 which represents supreme functionality. This means that Freundlich model has primacy in these adsorption isotherm studies and also suggests that the fabric yet not saturated with dye.

\section{Conclusion}

Red RR and Blue RR had good and almost uniform exhaustion\%. But exhaustion\% of Yellow RR was decreasing with the increase of shade\%. Fixation\% of Red RR was increasing with the increase of shade\%. The other two dyes' fixation was comparatively low. The difference in their fixation was significant in deeper concentrations. Washing had a considerable effect on Blue RR than other two, especially in case of hot wash. In the isotherm analysis, it was found that all of the three dyes had better fitted with Freundlich isotherm with almost linear model. Yellow RR had the better capacity for adsorption but due to its higher heterogeneity level its outcome was not good.

To get the best results from three of the dyes in a ternary shade matching, amount of electrolytes should be increased in dye bath especially for deeper concentrations, which can increase the affinity of dye to the fabric. As Yellow RR and Blue RR dyes are from VS group with higher reactivity, and found with better adsorption capacity in isotherm analysis; hydrolysis is the reason for their comparatively low fixation. Again Blue RR was found very sensitive to hot wash; more specifically in a temperature like $60^{\circ} \mathrm{C}$ which was actually the dyeing temperature also. So decreasing the alkali amount in dye bath and reducing the temperature from $60^{\circ} \mathrm{C}$ can be a good solution for increasing the compatibility of these dyes.

\section{Competing interests}

The authors declare that they have no competing interests.

\footnotetext{
Author details

${ }^{1}$ Daffodil International University, Dhaka, Bangladesh. ${ }^{2}$ Dhaka University of Engineering \& Technology, Gazipur, Bangladesh. ${ }^{3}$ BGMEA University of Fashion \& Technology, Dhaka, Bangladesh.
}

Received: 6 October 2014 Accepted: 2 January 2015

Published online: 19 February 2015

References

Ali, S, Khatri, A, Peerzada, HM, \& Ahmed, A. (2012). Effect of causticization on colour strength ( $\mathrm{k} / \mathrm{s}$ ) of reactive dyed lyocell fabric. Sci Int, 24(1), 51-54.

Ara, JN, Hasan, AM, Rahman, AM, Salam, AM, Salam, A, \& Alam, SMA. (2013). Removal of remazol red from textile waste water using treated sawdust - an effective way of effluent treatment. Bangladesh Pharmaceutical Journal, 16(1), 93-98. Broadbent, DA. (2001). Basic principles of textile coloration. Society of Dyers and Colourists, 332-348.

Cantrell, AC. (2008). Technical note: review of methods for linear least-squares fitting of data and application to atmospheric chemistry problems. Atmospheric Chemistry and Physics, 8, 5477-5487.

Cay, A, Atav, R, \& Duran, K. (2007). Effects of warp-weft density variation and fabric porosity of the cotton fabrics on their colour in reactive dyeing. Fibres \& Textiles in Eastern Europe, 15-1(60), 91-94.

Chinta, K. S. \& Vijaykumar, S. (2013). Technical facts \& figures of reactive dyes used in textiles. I.J.E.M.S 4(3), 308-312.

Choudhury, RKA. (2006). Textile preparation and dyeing (p. 387). New Delhi, India: Oxford and IBH Publishing Ltd.

Dalal, MM, \& Desai, RK. (1996). Dyeing effects of bi-functional reactive dyes on knitted cotton fabrics. American Dyestuff Reporter, April, 22-25.

Foo, YK, \& Hameed, HB. (2010). Insights into the modeling of adsorption isotherm systems. Chemical Engineering Journal, 156, 2-10.

Fowler, P. (1997). New trichromatic system for enhanced dyeing by the exhaust process. American Dyestuff Reporter, March, 62-66.

Shenai, AV. (1993). Technology of textile processing volume ii: chemistry of dyes and principles of dyeing. Sevak Publications, 495. 
Shenai, AV. (1997). Technology of textile processing volume vi: technology of dyeing (pp. 260-291). Gujarat, India: Sevak Publications.

Son, AY, Hong, PJ, Lim, TH, \& Kim, KT. (2005). A study of hetero bi-functional reactive dyes on nylon fibers: dyeing properties, dye moiety analysis and wash fastness. Dyes and Pigments, 66(3), 231-239.

Sultana, R. \& Uddin, Z. M. (2007). Compatibility testing of reactive dyes. Journal of Mechanical Engineering, ME38, 61-64.

Taylor, R. (1990). Interpretation of the correlation coefficient: a basic review. Journal of Diagnostic Medical Sonography, 6, 35-39.

Submit your manuscript to a SpringerOpen ${ }^{\circ}$ journal and benefit from:

- Convenient online submission

- Rigorous peer review

- Immediate publication on acceptance

- Open access: articles freely available online

- High visibility within the field

- Retaining the copyright to your article

Submit your next manuscript at $\gg$ springeropen.com 